\section{A) Check for updates}

Cite this: Polym. Chem., 2022, 13, 139

Received 6th November 2021, Accepted 27th November 2021 DOI: 10.1039/d1py01497h rsc.li/polymers

\title{
On the photopolymerization of mevalonic lactone methacrylate: exposing the potential of an overlooked monomer $\dagger$
}

\author{
Cansu Esen, Markus Antonietti (D) and Baris Kumru (DD * \\ Functional polymers remain at the core of polymer science in the second century of macromolecular \\ material research. Lactones are known as monomers for ring-opening polymerizations; however, poly- \\ mers containing lactone rings as pendant groups are rare. In this study, we revive an overlooked \\ monomer, mevalonic lactone methacrylate, by demonstrating its radical polymerization, reporting the \\ properties of the polymer product, and providing information on the thermal, UV, and hydrolytic stabilities \\ of both the monomer and polymer. Controlled polymerization (via RAFT) provides synthetic precision and \\ active terminal groups; thus, the formation of a simple block copolymer based on the aforementioned \\ polymer is presented. The pendant ring accepts nucleophilic attacks, so the initially hydrophobic polymer \\ can be altered to make it hydrophilic via reactions with nucleophiles in just seconds. Thus, the polymer \\ film provides responsive surface formation.
}

\section{Introduction}

In the second century since the first article reporting macromolecules, ${ }^{1}$ polymer chemistry continues to increase in importance. The polymer products developed within the last century have enabled our current lifestyle and technology, and the limits in this field are still being pushed further. Issues such as sustainability, ${ }^{2-4}$ recyclability ${ }^{5,6}$ and automated synthesis ${ }^{7-9}$ are examples of new questions and options. In parallel, the importance of employing photochemistry has been understood, and special attention has been paid to the development of novel photoinitiating systems. ${ }^{10-12}$ Furthermore, common polymerization techniques such as the free-radical, controlledradical, ionic, and step-growth mechanisms have been made available via either homogeneous ${ }^{13-15}$ or heterogeneous ${ }^{16-18}$ photoinitiating processes.

Searching for alternative monomers and polymers with novel functionalities is an obvious pathway for innovation. ${ }^{19,20}$ Many appealing technologies, such as 3D printing ${ }^{21,22}$ or polymerization-induced self-assembly (PISA) ${ }^{23}$ are constantly searching for alternative polymers. In this context, mevalonolactone is an overlooked starting product with interesting functions (Scheme $\mathrm{S} 1 \dagger$ ). Mevalonolactone (MVL) is the dehydration product of mevalonic acid; however, it immediately transforms

Max Planck Institute of Colloids and Interfaces, Department of Colloid Chemistry, Am Mühlenberg 1, 14424 Potsdam, Germany. E-mail: baris.kumru@mpikg.mpg.de $\dagger$ Electronic supplementary information (ESI) available. See DOI: 10.1039/ d1py01497h back into mevalonic acid in the presence of water. Both MVL and mevalonic acid are sustainable, sugar-based molecules and exhibit stereochemistry. Mevalonic acid is a key intermediate in the biological synthesis of molecules such as cholesterol (via the mevalonate pathway). ${ }^{24}$ Mevalonic acid is transformed into isopentenyl 5-pyrophosphate by enzymes, and thus starts the synthesis of terpene and steroid-like molecules. ${ }^{25}$

However, there are not many reports of the polymerization of MVL. In 2001, Ouchi and co-workers reported the one-pot ring opening copolymerization of MVL with L-lactide (10:90\% molar ratio) catalyzed by tin 2-ethylhexanoate or distannoxane to obtain branched biodegradable polymers (Scheme S2a $\dagger$ ). ${ }^{26}$ In this scenario, MVL is reported to behave as an initiator for L-lactide, as it possesses hydroxy groups; however, the copolymerization was reported to proceed very slowly (4 days). Zhang and colleagues prepared (-)- $\beta$-methyl- $\delta$-valerolactone from glucose through anhydro-MVL formation via a scalable biochemical route, which was then polymerized (ring-opening copolymerization) to obtain sustainable polymers (Scheme S2b $\dagger$ ). ${ }^{27,28}$ Chemical oxidations of MVL enable functionalization (via the opened ring) that can consecutively be employed for condensation polymerization, as disclosed in a patent. $^{29}$ Mevalonic lactone methacrylate (MVLMA) is another monomer that is obtained from the reaction of MVL with methacryloyl chloride (Scheme S2c $\dagger)^{30}$ and is commercially available, although at relatively high prices $(1 \mathrm{~g}: \sim 120$ euros). Only two articles (in 1999 and 2002) have reported MVLMA free-radical copolymerization with adamantane-containing monomers ( $\sim 56 \%$ conversion in 8 hours $)$ initiated by a 

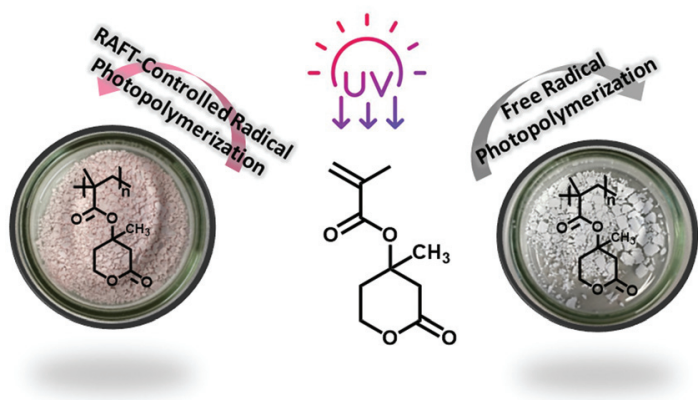

Scheme 1 The photo-induced radical polymerization of mevalonic lactone methacrylate (MVLMA).

thermal initiator, and the polymers were applied as laser resist materials. ${ }^{30,31}$ However, we found no reports on the homopolymerization of MVLMA or the properties of polyMVLMA.

In the present manuscript, we report the photopolymerization of mevalonic lactone methacrylate, analyze the polymers in detail, and post-functionalize the products. Controlled synthesis of the polymer by reversible additionfragmentation transfer (RAFT) agent is also described (Scheme 1).

\section{Experimental}

\section{Materials}

2,4,6-Trimethylbenzoyl diphenylphosphine oxide (TPO, 98\%, Sigma Aldrich), 2,2'-azobis(2-methylpropionitrile) (AIBN, \%98 Sigma Aldrich), 4-methyl-2-oxotetrahydro- $2 H$-pyran-4-yl methacrylate (MVLMA, $>98.0 \%$, stabilized with MEHQ, TCI), 1-methyl-2-pyrrolidinone (NMP, ACS grade, 99.0+\%, Alfa Aesar), 1-thioglycerol (97\%, Sigma Aldrich), ( \pm )-mevalonolactone (MVL, 97\% titration, Sigma Aldrich), cadmium sulfide (powder, Sigma Aldrich), ethyl 2-(phenylcarbonothioylthio)-2phenylacetate dithiobenzoate (RAFT agent, 98\%, Sigma Aldrich), deuterochloroform $\left(\mathrm{CDCl}_{3}-\mathrm{d} 6,99.8\right.$ atom\% D, Sigma Aldrich), dimethylsulfoxide-d6 (DMSO-d6, 99.9 atom\% D, Sigma Aldrich), deuterium oxide ( $\mathrm{D}_{2} \mathrm{O}, 99.9 \%$, Sigma Aldrich), hydrazine monohydrate (99+\%, Alfa Aesar), methanol $(\mathrm{MeOH}$, $\geq 99.9 \%$, Merck), phenylbis(2,4,6-trimethylbenzoyl)phosphine oxide (BAPOs, 97\%, Sigma Aldrich), styrene (St, $\geq 99 \%$, 4-tertbutylcatechol as stabilizer, Sigma Aldrich), and toluene (anhydrous 99.8\%, Sigma Aldrich) were obtained from commercial sources. St was passed through a basic alumina column prior to use to remove the inhibitor. Graphitic carbon nitride (CM) was freshly prepared according to a known literature procedure. ${ }^{16}$ Ultraviolet (UV) light irradiation was performed via a 30 W UV chip (Fdit 395-400 nm UV LED) connected to a custom-made circuit and a cooling system.

\section{Characterization}

X-ray diffraction (XRD) patterns of finely ground PMevL, RPMevL and PMevL- $b$-PS were obtained using a Bruker D8
Advance X-ray diffractometer $\mathrm{Cu}-\mathrm{K} \alpha(\lambda=0.154 \mathrm{~nm})$ equipped with a NaI scintillation counter-Scinti-Detector. The diffraction pattern was recorded in the $2 \theta$ range of $4-70^{\circ}$ with steps of $0.05^{\circ}$ and an acquisition time of $2 \mathrm{~s}$ per step. Fourier transform infrared (FT-IR) spectra were acquired on a Nicolet is 5 FT-IR spectrometer. Thermogravimetric analysis (TGA) was performed using a Thermo Microbalance TG 209 F1 Libra (Netzsch, Selb, Germany) under a nitrogen atmosphere with a heating rate of $10 \mathrm{~K} \mathrm{~min}^{-1}$ using an aluminum crucible for the samples. Differential scanning calorimetry (DSC) measurements were performed in aluminum pans as the reference using a Netzsch DSC204 system (Selb, Germany). The samples were heated from $35^{\circ} \mathrm{C}$ to $250^{\circ} \mathrm{C}$ and cooled to room temperature at a heating/cooling rate of $10{ }^{\circ} \mathrm{C} \mathrm{min}^{-1}$. Proton nuclear magnetic resonance $\left({ }^{1} \mathrm{H}-\mathrm{NMR}\right)$ and carbon nuclear magnetic resonance $\left({ }^{13} \mathrm{C}\right.$-NMR $)$ spectra were recorded at ambient temperature using a Bruker Ascend 400. Size exclusion chromatography (SEC) for PMevL, RPMevL, and PMevL- $b$-PS was conducted in $N$-methyl pyrrolidone containing $0.5 \mathrm{~mol} \mathrm{~L}^{-1} \mathrm{LiBr}$ and BSME as an internal standard using a column set consisting of two $300 \times 8 \mathrm{~mm}^{2}$ PSS-GRAM columns (particle size: $7 \mu \mathrm{m}$, porosity: 102 and $103 \AA$ at $70{ }^{\circ} \mathrm{C}$, flow rate: $0.8 \mathrm{~mL}$ $\min ^{-1}$ ) with a Shodex RI-71 detector. Calibration was carried out using poly(methyl methacrylate) (PMMA) standards from PSS.

Water contact angle measurement was performed using a Krüss G10 contact angle measuring system and recorded via the official Krüss software. The sample (a glass surface immersed in freshly prepared $20 \mathrm{mg}$ PMevL : $1 \mathrm{~mL} \mathrm{CHCI}_{3}$ solution and then placed in an oven set at $60{ }^{\circ} \mathrm{C}$ for $15 \mathrm{~min}$ ) with an ideally flat surface was placed in front of a camera which recorded droplets of water and hydrazine hydrate (1:5) and thioglycerol (1:5) aqueous solutions on the surface and estimated the angle between the droplet and the surface. This method is useful to determine surface properties, such as hydrophilicity and hydrophobicity in the present case.

\section{Comparative stability experiments of MVLMA and MVL}

$20 \mathrm{mg}$ of each substance was dissolved in $\mathrm{CDCl}_{3}$ and DMSOd6, placed in front of a UV light source at a distance of $60 \mathrm{~cm}$ under ambient conditions for 16 hours, and thereafter immediately analyzed via NMR. For a thermal comparison, $20 \mathrm{mg}$ of each substance was weighed out into glass vials and placed in an oven at $70^{\circ} \mathrm{C}$ overnight then analyzed via NMR.

\section{Free radical photopolymerization of mevalonic lactone methacrylate (PMevL)}

$0.5 \mathrm{~g}$ (2.5 mmol, 1 eq.) of MVLMA was weighed out into a round bottom flask containing $2 \mathrm{ml}$ toluene, followed by the addition of $2 \mathrm{mg}$ ( $0.0057 \mathrm{mmol}, 2.28 \mathrm{meq})$ TPO. The mixture was capped and purged with nitrogen for 15 minutes and then placed in front of a $30 \mathrm{~W}$ UV light source (60 cm distance) with continuous stirring for 16 hours at room temperature. Turbidity was observed within 3 minutes after switching on the light source. At the end of 16 hours, the mixture was precipitated in $20 \mathrm{~mL}$ methanol, filtered and left in a vacuum oven 
at $40{ }^{\circ} \mathrm{C}$ overnight (white powder, yield $\left.=57 \%\right)\left(M_{\mathrm{n}, \mathrm{SEC}}=29 \mathrm{~kg}\right.$ $\mathrm{mol}^{-1}, D=2.2$ ). The same procedure was performed employing BAPO as the photoinitiator instead of TPO, and the reaction proceeded under visible light irradiation for 24 hours. The resulting polymer is referred to as BPMevL (white powder, yield $=63 \%)\left(M_{\mathrm{n}, \mathrm{SEC}}=50 \mathrm{~kg} \mathrm{~mol}{ }^{-1}, Ð=3.22\right)$.

\section{RAFT-controlled radical photopolymerization of mevalonic lactone methacrylate (RPMevL)}

$0.5 \mathrm{~g}$ MVLMA was weighed out into a round bottom flask containing $2 \mathrm{ml}$ toluene, followed by the addition of $2 \mathrm{mg}$ TPO and $10 \mathrm{mg}$ RAFT agent. The mixture was capped and purged with nitrogen for 15 minutes and then placed in front of a 30 W UV light source (60 cm distance) with continuous stirring for 10 hours at room temperature. The initial reaction mixture had a transparent pale pink color, and turbidity was observed within 20 minutes after switching on the light source. At the end of 16 hours, the mixture was precipitated in $25 \mathrm{~mL}$ methanol, filtered, and left in a vacuum oven at $40{ }^{\circ} \mathrm{C}$ overnight (pale pink powder, yield $=20 \%)\left(M_{\mathrm{n}, \mathrm{SEC}}=7.5 \mathrm{~kg} \mathrm{~mol}^{-1}, Ð=\right.$ 1.55).

\section{RAFT-controlled block copolymer synthesis of PMevL (PMevL- $b$-PS)}

$0.02 \mathrm{~g}$ PMevL was weighed out into a round bottom flask containing $500 \mu \mathrm{L}$ NMP followed by the addition of $1 \mathrm{mg}$ TPO and $200 \mu \mathrm{L}$ styrene. The mixture was capped and purged with nitrogen for 15 minutes and then placed in front of a $30 \mathrm{~W}$ UV light source (60 cm distance) with continuous stirring for 6 hours at room temperature. The initial reaction mixture had a pale pink color, and after switching on the light source, the color transformed to bright yellow within 5 minutes. Approximately 3 hours later, the bright yellow color vanished and the mixture became transparent. The mixture was precipitated in methanol to provide a white powder, which was washed, filtered, and then left in a vacuum oven at $40{ }^{\circ} \mathrm{C}$ overnight (white powder, yield $=14 \%)\left(M_{\mathrm{n}, \mathrm{SEC}}=12.6 \mathrm{~kg} \mathrm{~mol}^{-1}, D=1.53\right)$.

\section{Ring-opening (pendant mevalonic lactone groups on PMevL) via hydrazine monohydrate}

$10 \mathrm{mg}$ PMevL was dispersed in $400 \mu \mathrm{L}$ distilled water. Subsequent step-wise ( 4 portions, each $100 \mu \mathrm{L}$ ) addition of hydrazine resulted in the formation of a clear solution, which was denoted as RO-PMevL. The solution was placed in an oven at $80{ }^{\circ} \mathrm{C}$ overnight and then left in a vacuum oven at $40{ }^{\circ} \mathrm{C}$ for 4 hours for the complete evaporation of water and residual hydrazine.

\section{Results and discussion}

Before attempting the polymerization experiments, determining the monomer stability was a prime concern. It is well known that mevalonolactone, as well as MVLMA, must be stored in the freezer in dark bottles. For this reason, we suspected poor structural stability against UV light irradiation and heat exposure. The monomer in $\mathrm{CDCl}_{3}$ and d-DMSO was kept under UV irradiation for 16 hours, and based on the collated ${ }^{1} \mathrm{H}-\mathrm{NMR}$ and ${ }^{13} \mathrm{C}-\mathrm{NMR}$ data, it was found to be stable under these conditions (Fig. S1 and S2 $\dagger$ ). Thermal stability was confirmed by placing the sample in an oven at $70{ }^{\circ} \mathrm{C}$ overnight, after which no structural changes were found (Fig. S3 $\dagger$ ). The surprising stability of the MVLMA monomer led us to investigate MVL again, and under comparable experimental conditions, both UV light irradiation and heat exposure affected its stability (Fig. S4 $\dagger$ ). One can conclude that destabilization mechanisms operate via the open alcohol group, and the modification via methacrylate addition to mevalonolactone to form the monomer MVLMA provides stability to the structure.

\section{Free radical photopolymerization}

Photo-induced radical polymerization of MVLMA was performed as described in the experimental part. In brief, MVLMA (monomer) and TPO (photoinitiator) were dissolved in toluene and purged with nitrogen, and then placed in front of a UV light source under continuous stirring for 16 hours. Rapid turbidity in the initially transparent reaction medium was observed within 3 minutes after switching on the light source. The fast development of turbidity led us to consider possible precipitation polymerization; however, a quick check via light microscopy did not show particle formation. Based on visual observations, the turbidity and viscosity slightly increased over time, and at the end of 16 hours, a white powder, which was denoted as PMevL, was successfully obtained after precipitation in $\mathrm{MeOH}$ followed by a filtration and drying process in $58 \mathrm{wt} \%$ solid yield (Table 1). The ${ }^{1} \mathrm{H}-\mathrm{NMR}$ and ${ }^{13} \mathrm{C}-\mathrm{NMR}$ results confirmed polymer formation based upon the disappearance of the vinylic hydrogen signals of the methacrylate group on MVLMA $\left(\mathrm{CH}_{2}=\mathrm{C}-\right.$, a $)$ while new signals characteristic of the polymer backbone of PMevL appeared (Fig. 1 and Fig. $\mathrm{S} 5 \dagger$ ).

In accordance with the NMR results, the FT-IR spectrum of PMevL did not exhibit a $\mathrm{C}=\mathrm{C}$ stretching vibration at $1637 \mathrm{~cm}^{-1}$. Additionally, the two $\mathrm{C}=\mathrm{O}$ stretching vibrations of MVLMA, which are located at $1741 \mathrm{~cm}^{-1}$ and $1701 \mathrm{~cm}^{-1}$ as a result of the lowered stretching frequency related to the conjugation between the $\mathrm{C}=\mathrm{C}$ and $\mathrm{C}=\mathrm{O}$ bonds, transformed into one signal at $1717 \mathrm{~cm}^{-1}$ after the polymerization (Fig. 2a). The time-dependent molecular weight evolution of PMevL was demonstrated via SEC (Fig. $2 \mathrm{~b}$ and Table 1). The first sample $(t$ $=1$ ) was taken 6 hours after the starting time, and subsequent samples were taken every 2 hours until the reaction period was complete (16 hours). As can be seen from Fig. $2 b$, the efficient formation of large macromolecules took place after 10 hours. According to the PMMA calibration, PMevL exhibited a relatively low $M_{\mathrm{n}, \mathrm{SEC}}$ value of $29 \mathrm{~kg} \mathrm{~mol}{ }^{-1}$ with a slightly broad molecular mass distribution with a $D$ of 2.2. Similar molar mass values and polymerization kinetics were obtained in the literature (for random copolymerization with 2-methyl-2-adamantane $)^{30}$ as well, which can be explained as arising from steric hindrance. To investigate the hydrolytic stability of the polymer, we immersed the polymer in $\mathrm{D}_{2} \mathrm{O}$ for 2 days, then fil- 
Table 1 SEC results from the as-synthesized polymers in combination with the yield and reaction conditions

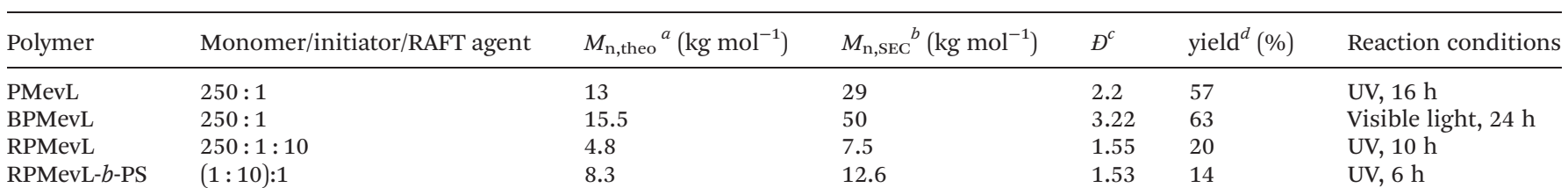

${ }^{a}$ Calculated as described in the Experimental section. ${ }^{b}$ Determined via SEC. ${ }^{c}$ Obtained via SEC. ${ }^{d}$ Calculated gravimetrically.

a)

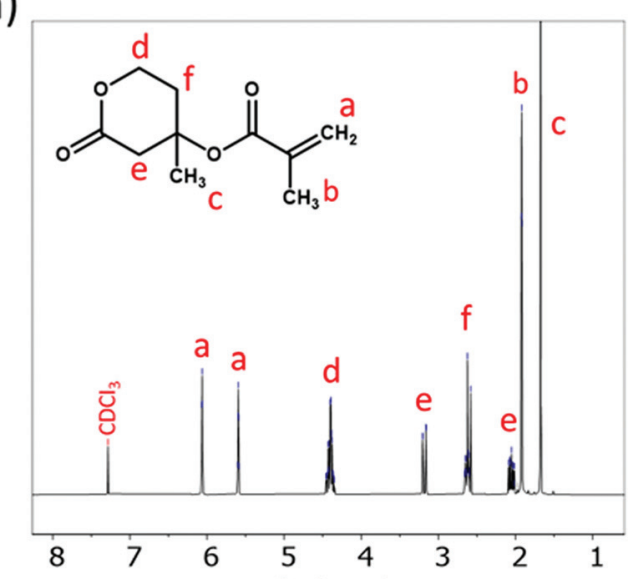

b)

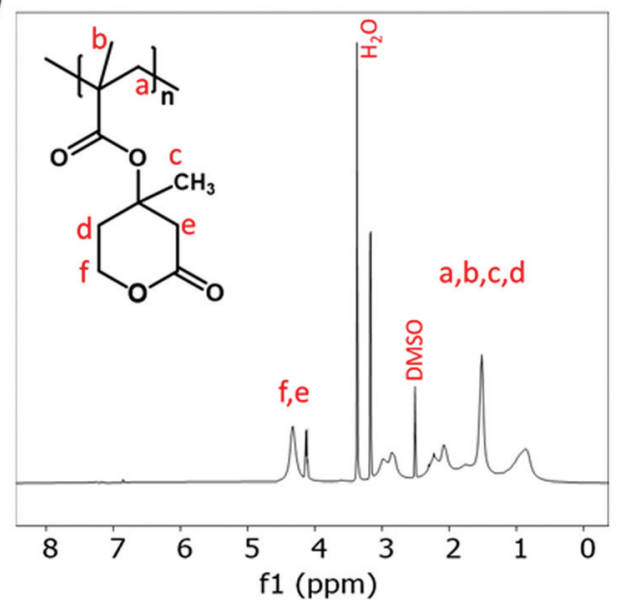

Fig. $1{ }^{1} \mathrm{H}-$ NMR spectra of MVLMA (a) and PMevL (b).

tered it and obtained its ${ }^{1} \mathrm{H}$-NMR spectrum; however, no dissolved product was found, which indicates the stability of the mevalonolactone pendant group against spontaneous hydrolysis (data not shown). The mevalonolactone ring might inhibit significant chain growth.

The thermal properties of PMevL were investigated via TGA and DSC. The TGA measurements showed a two-step degradation profile; the step from $186^{\circ} \mathrm{C}$ to $400{ }^{\circ} \mathrm{C}$ was attributed to MVL ring decomposition and depolymerization with $62.7 \mathrm{wt} \%$ weight loss, and the second degradation step was attributed to final fragmentation and extended to $450{ }^{\circ} \mathrm{C}$ with $30.7 \mathrm{wt} \%$ mass change (Fig. 3a). DSC measurement showed a single

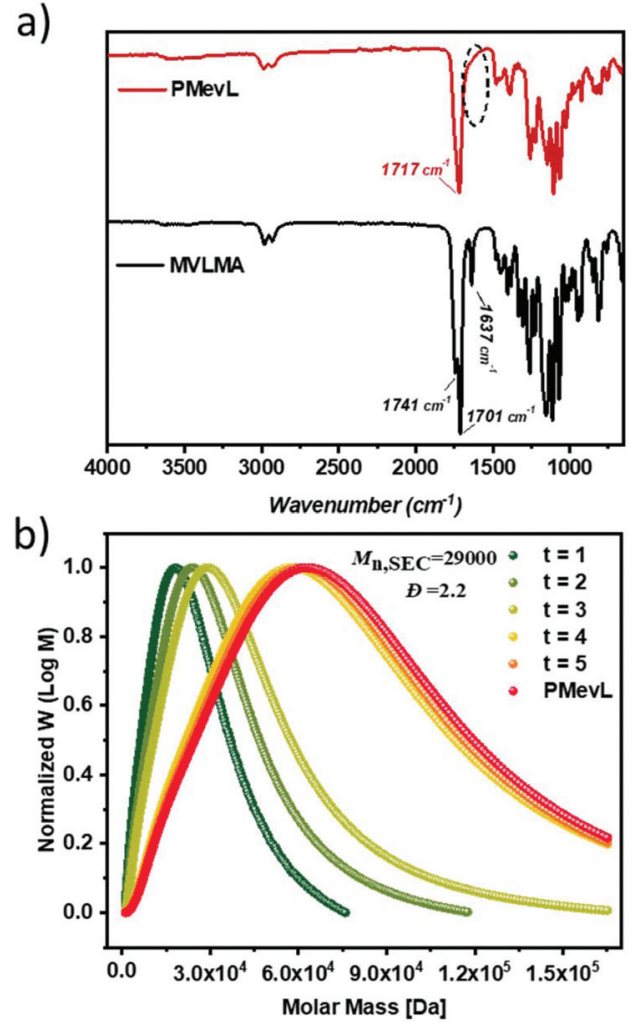

Fig. 2 FT-IR spectra of MVLMA and PMevL (a), and the kinetics of $\mathrm{PMevL}$ with respect to the normalized number-average molecular weight obtained via SEC (b).

point, a relatively high $T_{\mathrm{g}}$ at $154{ }^{\circ} \mathrm{C}$, followed by structural decomposition with gradually increasing temperature (Fig. 3b).

In addition, photopolymerization was attempted in the visible-light range using BAPO as a radical photoinitiator. Our efforts to conduct photoredox-type visible-light-induced polymerization by employing cadmium sulfide and carbon nitride semiconductors failed to produce clean polymers, which might be ascribed to photogenerated oxidative species influencing the MVL ring stability. On the other hand, BAPObased initiation via visible light resulted in an $M_{\mathrm{n}, \mathrm{SEC}}$ value of $50 \mathrm{~kg} \mathrm{~mol}^{-1}$ with a $D$ of 3.22 , and polymer structures were confirmed via both FT-IR and NMR analysis (BPMevL) (Fig. S6†). It is conclusive that mevalonic lactone methacrylate can be successfully photopolymerized via visible light as well. However, the dispersity index of BPMevL, which exhibited a much higher value than that of PMEvL, is not desirable when 

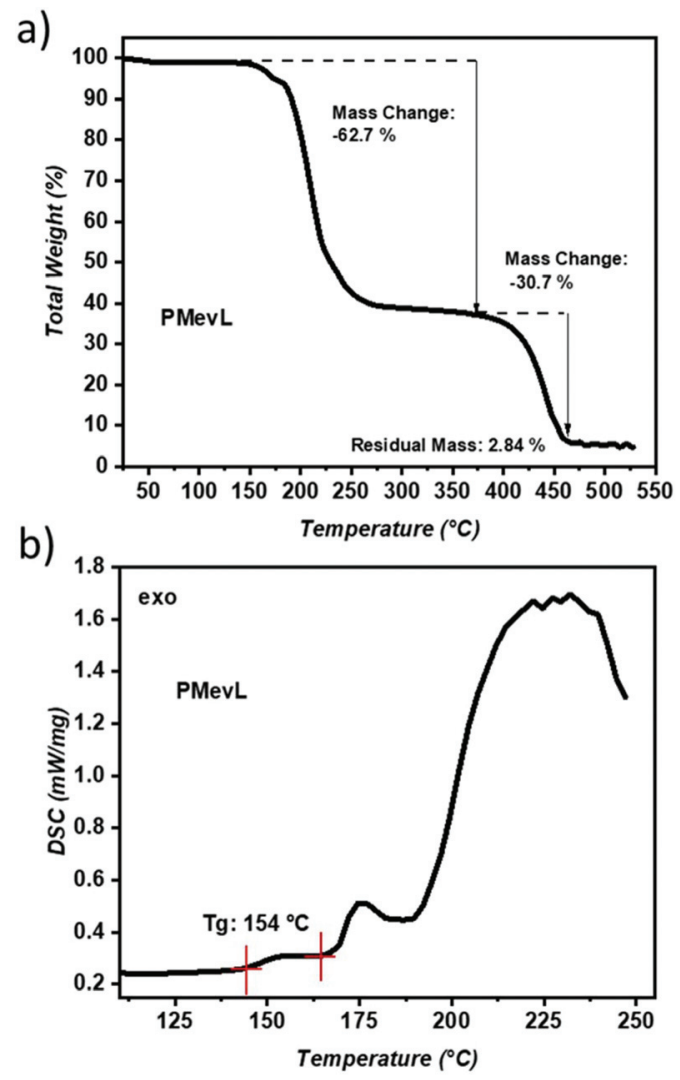

Fig. 3 TGA measurements (a) and DSC measurements (b) of PMevL.

its further utilization is considered. Therefore, PMevL was chosen for the subsequent post-modification studies.

\section{Nucleophilic ring-opening of pendant mevalonolactone units}

The obtained polymer products with the mevalonic lactone pendant groups were then explored for further functionalization. Simple ring-opening under mild reaction conditions was realized via nucleophilic attack of hydrazine hydrate on the carbonyl carbon, which leads to the opening of the ring and forms hydrazide groups (Scheme S3†). As can be seen from Fig. S7, $\dagger$ PMevL is inherently hydrophobic (water-dispersible). Nevertheless, after the step-wise addition of hydrazine, an immediate phase transition towards a clear solution was observed within a couple of seconds, which confirmed the effective ring opening. Afterwards, the as-prepared polymer was purified by removing the residual hydrazine and water via evaporation as described in the Experimental section (RO-PMevL). According to ${ }^{1} \mathrm{H}$-NMR and ${ }^{13} \mathrm{C}-\mathrm{NMR}$ results, the $\alpha$ hydrogens of mevalonic lactone were shielded from $4.1 \mathrm{ppm}$ to 3.5 ppm (Fig. S8a $\dagger$ ). Moreover, the FT-IR spectrum exhibited typical N-H stretching of primary amine and secondary amide groups at $3270 \mathrm{~cm}^{-1}, 2931 \mathrm{~cm}^{-1}$, and $2628 \mathrm{~cm}^{-1}$, as well as inplane $\mathrm{N}-\mathrm{H}$ bending at $1550 \mathrm{~cm}^{-1}$ (Fig. S8 $\mathrm{c}^{\dagger}$ ).

This responsive functionality has high potential for further applications. To gain some insight into this, PMevL was dissolved in chloroform (20 mg:1 mL) and coated on a glass
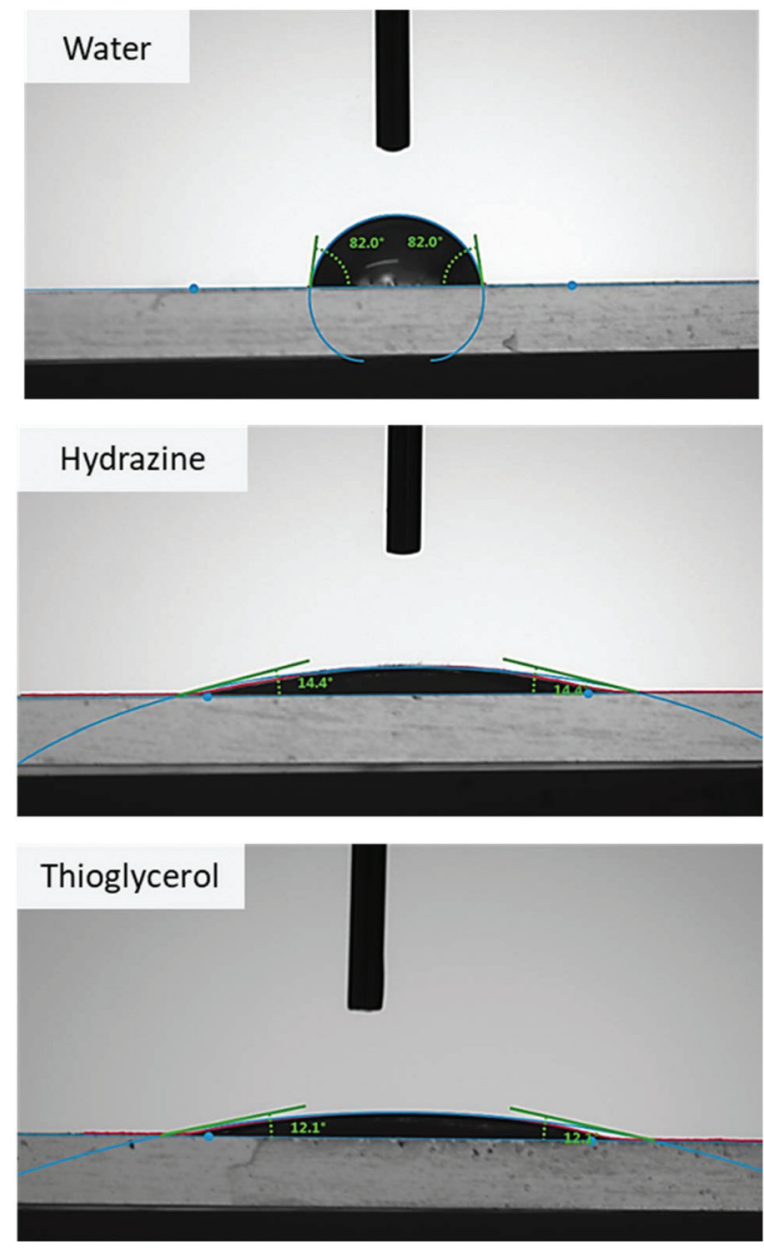

Fig. 4 Contact angle results for the PMevL film coated on glass in response to water, hydrazine, and thioglycerol.

surface; it formed good quality films, similar to polymethacrylates. Subsequently, water contact angle measurements (WCA) were performed, in both distilled water and in the presence of nucleophilic molecules. As can be seen in Fig. 4, distilled water gives a WCA of $82^{\circ}$, indicating hydrolytic stability as well as the hydrophobic nature of PMevL. On the other hand, when strong nucleophiles such as hydrazine and thioglycerol are present, the significant decrease in the WCA results can be attributed to hydrolysis based on the pendant MVL groups on the PMevL-coated surface $\left(14.4^{\circ}\right.$ and $12.1^{\circ}$, respectively). This further confirms the ease of ring-opening via nucleophiles, which does not require physical stimuli like manual shaking, but also can occur over an immobile platform over time. In summary, PMevL polymer films are responsive surfaces and can be employed in many potential applications spanning from sensing to molecule transport.

\section{RAFT-controlled photopolymerization}

Controlled radical polymerization is very useful when low dispersities and/or block copolymer formation are required. ${ }^{32-34}$ In this part, we employ a dithiobenzoate RAFT agent for the 
Table 2 Solubility data for the as-synthesized polymers

\begin{tabular}{|c|c|c|c|c|c|c|c|c|c|}
\hline PMevL & $x$ & $x$ & $\checkmark$ & $\checkmark$ & $\checkmark$ & $\times$ & $x$ & $x$ & $\checkmark$ \\
\hline BPMevL & $x$ & $x$ & $\checkmark$ & $\checkmark$ & $\checkmark$ & $x$ & $x$ & $x$ & $\checkmark$ \\
\hline RPMevL- $b$-PS & $x$ & $x$ & $\checkmark$ & $\checkmark$ & $\checkmark$ & $x$ & $x$ & $\sim$ & $\checkmark$ \\
\hline
\end{tabular}

$\times$ : insoluble, $\sim$ : slightly soluble $\checkmark$ : soluble.

synthesis of PMevL as described in the Experimental section. Unlike the free-radical photopolymerization, the RAFTmediated/controlled analogue (RPMevL) was terminated in 10 hours (instead of 16 hours) and after purification resulted in a pale pink colored powder, which indicates the presence of RAFT end-groups on the resulting polymer chains (still dormant polymer chain). This polymer can be used as a macro-chain transfer agent for block copolymer synthesis with styrene by employing freshly prepared RPMevL under UV light. The resulting white powder copolymer with $14 \mathrm{wt} \%$ solid yield is denoted as RPMevL-b-PS (Table 1). At this point, a solubility chart of the synthesized polymer is provided, which aims to guide researchers employing these polymers in future (Table 2). In addition, digital images of the copolymerization visually present the reaction progress; the initial pale pink color that represents RAFT end groups then transforms to bright yellow within 3 minutes due to the exposure to UV light, and 3 hours later becomes totally transparent (Fig. S9†).

${ }^{1} \mathrm{H}-\mathrm{NMR}$ confirmed successful polymerization, as the corresponding double-bond signals disappeared and the signals corresponding to the repeating groups of both RPMevL and RPMevL- $b$-PS grew (between 1-2 ppm). For the block copolymer, benzylic signals belonging to polystyrene were found (Fig. 5).

FT-IR spectra of RPMevL and RPMevL-b-PS in combination with PMevL clearly reveal the efficient block copolymer synthesis. Polystyrene incorporation was confirmed based on its characteristic signals, such as the signal at $3030 \mathrm{~cm}^{-1}$ corresponding to the aromatic $\mathrm{C}-\mathrm{H}$ stretching vibration, those at $1585 \mathrm{~cm}^{-1}$ and $1494 \mathrm{~cm}^{-1}$ corresponding to aromatic $\mathrm{C}-\mathrm{H}$ bond stretching and lastly the signal at $698 \mathrm{~cm}^{-1}$ corresponding to aromatic $\mathrm{C}-\mathrm{H}$ deformation vibration (Fig. 6a). a)

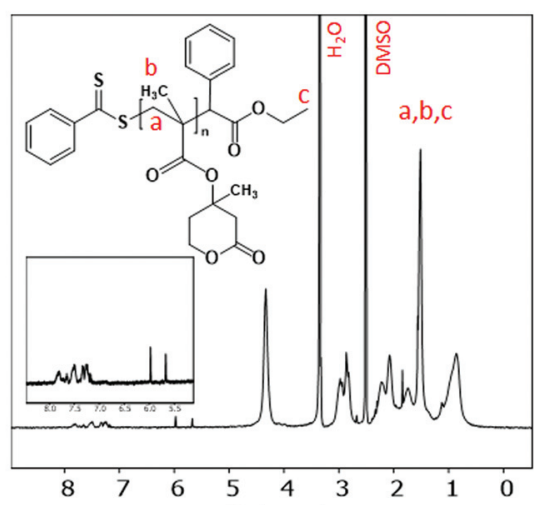

b)

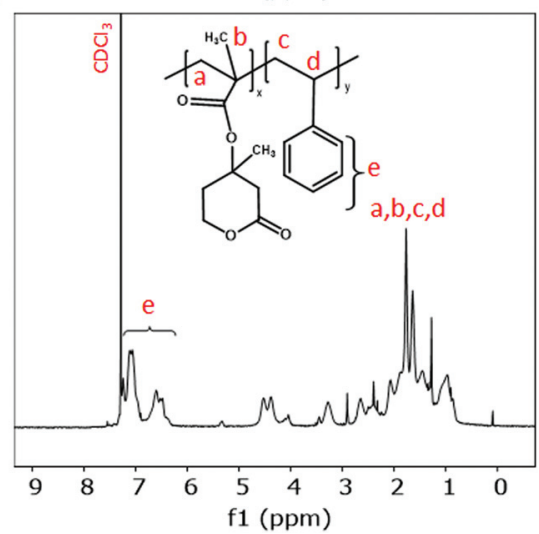

Fig. $5{ }^{1} \mathrm{H}-\mathrm{NMR}$ spectra of RPMevL (a) and RPMevL-b-PS (b).

a)

b)
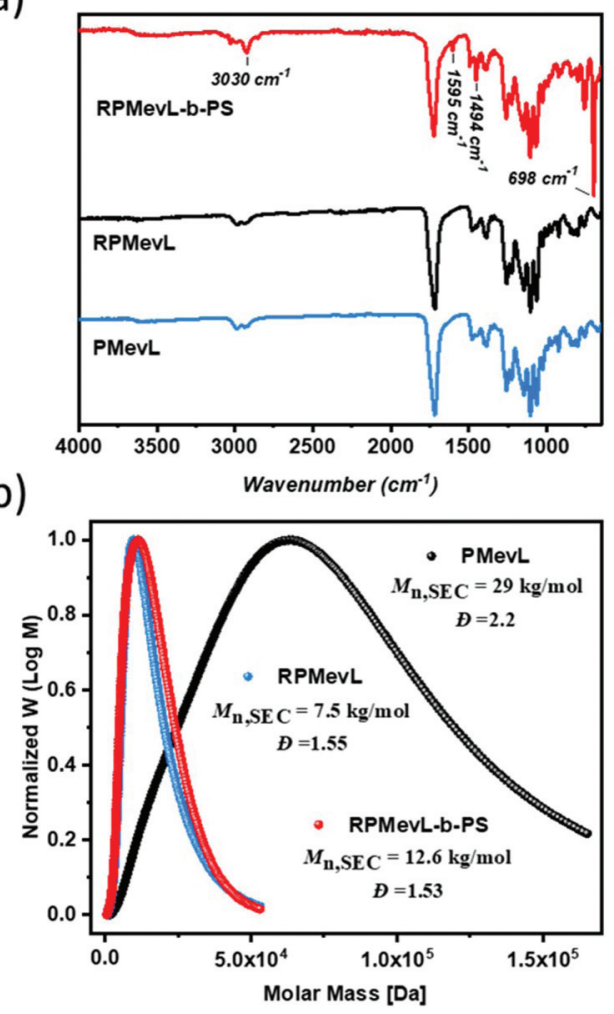

Fig. 6 FT-IR spectra (a) and normalized SEC results depicting the molar mass distributions (b) of PMevL, RPMevL, and RPMevL- $b$-PS. 
a)

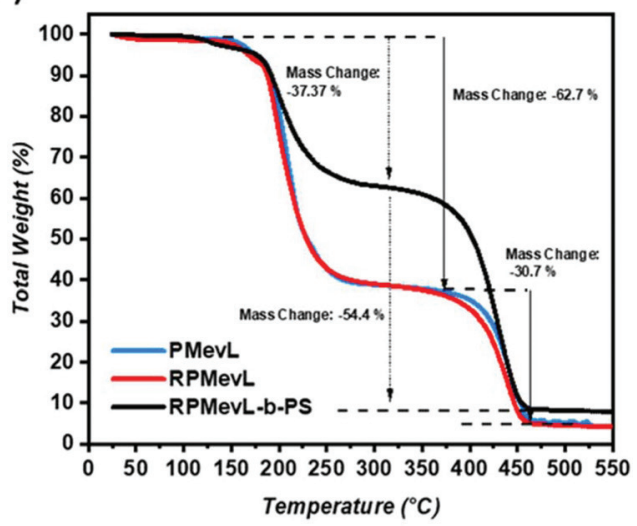

b)

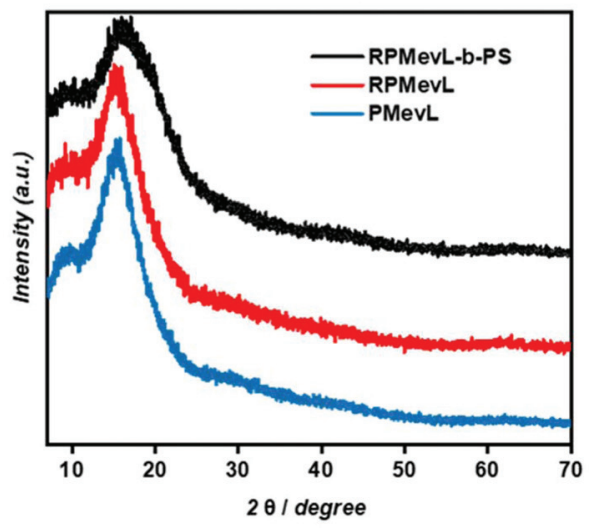

Fig. 7 TGA measurements (a) and XRD profiles (b) of PMevL, RPMevL, and RPMevL-b-PS.

A narrow molecular mass distribution with low $Ð$ was expected from the RAFT-mediated product. As shown in Fig. 6b, the controlled photopolymerization of RPMevL first resulted in an $M_{\mathrm{n}, \mathrm{SEC}}$ value of $7.5 \mathrm{~kg} \mathrm{~mol}^{-1}$ with a $D$ of 1.55 , while its co-polymer (RPMevL- $b$-PS) exhibited an $M_{\mathrm{n}, \mathrm{SEC}}$ value of $12.6 \mathrm{~kg} \mathrm{~mol}^{-1}$ with a $Ð$ of 1.53. The effect of employing the RAFT agent on the molar mass distribution is clearly observed by comparison to PMevL $\left(M_{\mathrm{n}, \mathrm{SEC}}\right.$ value of $29 \mathrm{~kg} \mathrm{~mol}^{-1}$ with $\left.D=2.2\right)$, and block copolymer formation could be obtained as a result of the RAFT chemistry.

The thermal degradation profiles of all polymers are in accordance with the expectations (Fig. 7a). The enhanced thermal stability of RPMevL- $b$-PS is attributed to block copolymer formation with styrene units, and this polymer yields $10 \%$ carbonaceous material at $450{ }^{\circ} \mathrm{C}$, which is a higher value compared to those of the homopolymers. However, a similar step-wise degradation profile was obtained. Due to the lactone unit, the possible crystal structures of the thus-formed polymers were investigated via powder XRD. However, strong amorphous regions with broad signals at $2 \theta=15.2$ and 15.4 were detected for all polymers, with no clear indication of crystallinity (Fig. 7b).

\section{Conclusions}

We described the photo-induced radical homopolymerization of mevalonic lactone methacrylate, including a systematic ana- lysis of the monomer and mevalonolactone stabilities. The polymerization behavior of the monomer was investigated using photoinitiation, and the final properties of the polymer product were described. The $T_{\mathrm{g}}$ value $\left(154{ }^{\circ} \mathrm{C}\right)$ of the polymer was rather high. As the lactone groups of the thus-formed polymers are susceptible to modification, nucleophilic ring opening with hydrazine was demonstrated to transform the hydrophobic polymer into a hydrophilic analogue in seconds, and homogeneous polymer films exhibit responsive surface properties. Furthermore, controlled photopolymerization via a RAFT agent provides active end groups to the polymer, and the chain extension abilities (block copolymer formation) of the RAFT-terminated polymer were confirmed. Lactone groups are known as monomers; however, pendant lactone units in polymer chains are rare. Thus, MVLMA polymers constitute an attractive avenue for researchers. The intention of this manuscript is to motivate researchers to rediscover the MVLMA monomer and its polymer properties from this perspective.

\section{Conflicts of interest}

There are no conflicts to declare.

\section{Acknowledgements}

Open Access funding provided by the Max Planck Society.

\section{References}

1 H. Staudinger, Ber. Dtsch. Chem. Ges., 1920, 53, 1073-1085.

2 M. Al-Naji, H. Schlaad and M. Antonietti, Macromol. Rapid Commun., 2021, 42, 2000485.

3 D. E. Fagnani, J. L. Tami, G. Copley, M. N. Clemons, Y. D. Y. L. Getzler and A. J. McNeil, Macromolecules, 2021, 10, 41-53.

4 C. Veith, F. Diot-Néant, S. A. Miller and F. Allais, Polym. Chem., 2020, 11, 7452-7470.

5 E. Gabirondo, A. Sangroniz, A. Etxeberria, S. Torres-Giner and H. Sardon, Polym. Chem., 2020, 11, 4861-4874.

6 J. G. Kim, Polym. Chem., 2020, 11, 4830-4849.

7 M. Tamasi, S. Kosuri, J. DiStefano, R. Chapman and A. J. Gormley, Adv. Intell. Syst., 2019, 2, 1900126.

8 R. Hoogenboom, M. A. R. Meier and U. S. Schubert, Macromol. Rapid Commun., 2003, 24, 15-32.

9 G. R. D. Prabhu and P. L. Urban, Chem. Rev., 2020, 120, 9482-9553.

10 Y. Yagci, S. Jockusch and N. J. Turro, Macromolecules, 2010, 43, 6245-6260.

11 K. Sun, H. Chen, Y. Zhang, F. Morlet-Savary, B. Graff, P. Xiao, F. Dumur and J. Lalevée, Eur. Polym. J., 2021, 151, 110410.

12 H. Chen, G. Noirbent, Y. Zhang, K. Sun, S. Liu, D. Brunel, D. Gigmes, B. Graff, F. Morlet-Savary, P. Xiao, F. Dumur and J. Lalevée, Dyes Pigm., 2021, 188, 109213. 
13 X. Pan, M. A. Tasdelen, J. Laun, T. Junkers, Y. Yagci and K. Matyjaszewski, Prog. Polym. Sci., 2016, 62, 73-125.

14 H. C. Kiliclar, G. Yilmaz and Y. Yagci, Macromol. Rapid Commun., 2021, 42, 2000686.

15 A. Suerkan, E. A. Alkan, K. Kaya, Y. A. Udum, L. Toppare and Y. Yagci, Prog. Org. Coat., 2021, 154, 106189.

16 B. Kumru, V. Molinari, M. Hilgart, F. Rummel, M. Schaeffler and B. V. K. J. Schmidt, Polym. Chem., 2019, 10, 3647-3656.

17 Z. J. Wang, K. Landfester and K. A. I. Zhang, Polym. Chem., 2014, 5, 3559-3562.

18 H.-C. Lee, M. Fantin, M. Antonietti, K. Matyjaszewski and B. V. K. J. Schmidt, Chem. Mater., 2017, 29, 9445-9455.

19 A. S. Abd-El-Aziz, M. Antonietti, C. Barner-Kowollik, W. H. Binder, A. Böker, C. Boyer, M. R. Buchmeiser, S. Z. D. Cheng, F. D’Agosto, G. Floudas, H. Frey, G. Galli, J. Genzer, L. Hartmann, R. Hoogenboom, T. Ishizone, D. L. Kaplan, M. Leclerc, A. Lendlein, B. Liu, T. E. Long, S. Ludwigs, J. F. Lutz, K. Matyjaszewski, M. A. R. Meier, K. Müllen, M. Müllner, B. Rieger, T. P. Russell, D. A. Savin, A. D. Schlüter, U. S. Schubert, S. Seiffert, K. Severing, J. B. P. Soares, M. Staffilani, B. S. Sumerlin, Y. Sun, B. Z. Tang, C. Tang, P. Théato, N. Tirelli, O. K. C. Tsui, M. M. Unterlass, P. Vana, B. Voit, S. Vyazovkin, C. Weder, U. Wiesner, W. Y. Wong, C. Wu, Y. Yagci, J. Yuan and G. Zhang, Macromol. Chem. Phys., 2020, 221, 2000216.

20 J.-F. Lutz, ACS Macro Lett., 2020, 9, 185-189.

21 Y. Zhang, Y. Xu, A. Simon-Masseron and J. Lalevée, Chem. Soc. Rev., 2021, 50, 3824-3841.
22 E. M. Wilts, A. M. Pekkanen, B. T. White, I. Meenakshisundaram, D. C. Aduba-Jr., C. B. Williams and T. E. Long, Polym. Chem., 2019, 10, 1442-1451.

23 N. J. W. Penfold, J. Yeow, C. Boyer and S. P. Armes, ACS Macro Lett., 2019, 8, 1029-1054.

24 L. Gong, Y. Xiao, F. Xia, P. Wu, T. Zhao, S. Xie, R. Wang, Q. Wen, W. Zhou, H. Xu, L. Zhu, Z. Zheng, T. Yang, Z. Chen and Q. Duan, Cell Death Dis., 2019, 10, 327.

25 J. L. Goldstein and M. S. Brown, Nature, 1990, 343, 425430.

26 F. Tasaka, Y. Ohya and T. Ouchi, Macromol. Rapid Commun., 2001, 22, 820-824.

27 C. Zhang, D. K. Schneiderman, T. Cai, Y.-S. Tai, K. Fox and K. Zhang, ACS Sustainable Chem. Eng., 2016, 4, 4396-4402.

28 M. Xiong, D. K. Schneiderman, F. S. Bates, M. A. Hillmyer and K. Zhang, Proc. Natl. Acad. Sci. U. S. A., 2014, 111, 8357-8362.

29 D. Dugar and T. Friedberger, Polymers Prepared From Mevalonolactone And Derivatives, US20160130389A1, 2016.

30 R. Dammel, M. Cook, A. Klauck-Jacobs, T. Kudo, S. Mehtsun, J. Oberlander, M. Padmanaban and D. L. Rahman, J. Photopolym. Sci. Technol., 1999, 12, 433444.

31 K. Nozaki and E. Yano, Fujitsu Sci. Tech. J., 2002, 38, 3-12.

32 A. Plucinski, M. Pavlovic and B. V. K. J. Schmidt, Macromolecules, 2021, 54, 5366-5375.

33 B. Kumru and N. Bicak, RSC Adv., 2015, 5, 30936-30942.

34 F. Lauterbach, M. Rubens, V. Abetz and T. Junkers, Angew. Chem., Int. Ed., 2018, 57, 14260-14264. 